Bundesgesundheitsbl 2012 $55: 601-603$

DOI 10.1007/s00103-012-1481-8

Online publiziert: 21. April 2012

๑) Springer-Verlag 2012

\author{
H.-H. König ${ }^{1}$ S.N. Willich ${ }^{2}$ U. Koch-Gromus ${ }^{3}$ \\ ${ }^{1}$ Institut für Medizinische Soziologie, Sozialmedizin und Gesundheitsökonomie (IMSG), Hamburg \\ Center for Health Economics, Universitätsklinikum Hamburg-Eppendorf, Hamburg \\ ${ }^{2}$ Institut für Sozialmedizin, Epidemiologie und Gesundheitsökonomie, \\ Charité - Universitätsmedizin Berlin, Berlin \\ ${ }^{3}$ Institut und Poliklinik für Medizinische Psychologie, Zentrum für Psychosoziale \\ Medizin, Universitätsklinikum Hamburg-Eppendorf, Hamburg
}

\title{
Perspektiven der Gesundheitsökonomie in Deutschland
}

mit die begrenzten Mittel möglichst effizient eingesetzt werden. Ökonomische Probleme sind im Gesundheitswesen heute allgegenwärtig und werden auch von den Akteuren in der Gesundheitsversorgung zunehmend als solche wahrgenommen. Im Gegensatz zu früher, negiert heute kaum einer das für die Ökonomie zentrale Problem der Knappheit der Ressourcen, wenngleich Ursachen und Lösungsmöglichkeiten ökonomischer Probleme von den Akteuren häufig kontrovers und manchmal auch emotional diskutiert werden. Gesundheitsökonomie als Wissenschaft hat die Aufgabe, Sachlichkeit und Transparenz in solche Diskussionen zu bringen.

Im vorliegenden Schwerpunktheft werden anhand von 13 Beiträgen Einblicke in den Stand und die Perspektiven der gesundheitsökonomischen Forschung in Deutschland gegeben. Im einleitenden Beitrag skizziert V. Ulrich die Entwicklung der Gesundheitsökonomie in Deutschland und beschreibt deren wesentliche Themenfelder. Dabei grenzt er den Bereich der Ökonomie des Gesundheitswesens, der sich mit der ökonomischen Analyse des Gesundheitssystems befasst, vom Bereich der Ökonomie der Gesundheit ab, zu dem die gesundheitsökonomische Evaluation zählt. An dieser Einteilung orientiert sich auch die Auswahl der übrigen Beiträge für das Schwerpunktheft.
Demografischer Wandel und medizinisch-technischer Fortschritt führen zu fortschreitenden Ausgabensteigerungen und Finanzierungsproblemen im Gesundheitswesen, denen mit Maßnahmen zur Steigerung der Effizienz des Gesundheitssystems begegnet wird. Hierzu gehören beispielsweise der morbiditätsorientierte Risikostrukturausgleich zwischen den gesetzlichen Krankenkassen, die fallpauschalierte Vergütung für stationäre Krankenhausleistungen und erweiterte Möglichkeiten zur integrierten Versorgung. Diesen Themen aus dem Bereich der Ökonomie des Gesundheitswesens widmen sich vier Beiträge.

S. Felder geht der Frage nach, wie sich die steigende Lebenserwartung - eine der Komponenten des demografischen Wandels - auf zukünftige Gesundheitsausgaben auswirkt. Auf Grundlage von Krankenkassendaten und Bevölkerungsvorausberechnungen zeigt er, dass die Auswirkungen eher moderat sind, da der Anstieg der individuellen Gesundheitsausgaben nicht durch das Alter, sondern insbesondere durch die Nähe zum Tod verursacht wird. Werden diese „Sterbekosten " in Prognosen explizit berücksichtigt, ergibt sich ein geringerer demografisch bedingter Ausgabenanstieg als in Berechnungen, in denen lediglich altersspezifische Gesundheitsausgabenprofile verwendet werden. 
R. Jahn, S. Schillo und J. Wasem analysieren die Zielgenauigkeit des morbiditätsorientierten Risikostrukturausgleichs (Morbi-RSA) in der gesetzlichen Krankenversicherung. Dieser ersetzt seit dem Jahr 2009 den alten demografischen RSA, um morbiditätsbedingte Unterschiede in den Gesundheitsausgaben zwischen den Krankenkassen auszugleichen und damit im wettbewerblichen System der gesetzlichen Krankenversicherung Anreize zur Risikoselektion zu vermeiden. Die Autoren zeigen, dass der Morbi-RSA die zu erwartenden Leistungsausgaben wesentlich genauer vorhersagt als der alte demografische RSA. Sie weisen jedoch darauf hin, dass ein zielgenauer RSA zwar eine notwendige, aber keine hinreichende Voraussetzung für einen funktionalen Kassenwettbewerb darstellt.

A. Geissler, D. Scheller-Kreinsen, W. Quentin und R. Busse vergleichen Systeme zur diagnosebezogenen Klassifikation von Krankenhausleistungen in zwölf europäischen Ländern. Diagnosebezogene Fallgruppen (Diagnosis Related Groups - DRGs) werden insbesondere als Grundlage für die fallpauschalierte Vergütung eingesetzt, die Anreize zur Effizienzsteigerung in der Leistungserstellung setzen soll. Die Autoren zeigen auf, dass zwischen den Ländern erhebliche Unterschiede im Design der wesentlichen DRG-Systembausteine bestehen und diskutieren Herausforderungen für sich daraus ergebende zukünftige Entwicklungen.

A. Lange, S. Braun und W. Greiner befassen sich mit ökonomischen Aspekten der integrierten Versorgung, durch die Schnittstellenprobleme zwischen Versorgungsbereichen minimiert und mehr Wettbewerb erreicht werden soll. Sie zeigen, dass in Deutschland trotz erweiterter gesetzlicher Spielräume eine umfassende integrierte Gesundheitsversorgung nach wie vor auf wenige Leuchtturmprojekte beschränkt ist. Sie führen dies insbesondere auf zu geringe Anreize für die Anbieter und Nachfrager solcher Versorgungsformen zurück sowie auf spezifische Umsetzungshürden, zu denen insbesondere Finanzierungsprobleme gehören.

Der Beitrag von F. Breyer befasst sich mit der Rationierung von Gesundheitsleistungen und spricht sich für eine offe- ne Debatte über die Knappheit und Verteilung von Gesundheitsleistungen in Deutschland aus. Er hinterfragt die herkömmliche Verwendung des Begriffs der Rationierung als „Vorenthaltung notwendiger Leistungen “, stellt die Vor- und Nachteile verschiedener Rationierungsformen dar und diskutiert inhaltliche sowie prozedurale Fragen einer möglichen expliziten Rationierung in der gesetzlichen Krankenversicherung. Indem er die Kosteneffektivität von Gesundheitsleistungen als ein mögliches Rationierungskriterium diskutiert, schlägt der Beitrag von F. Breyer die Brücke zu den übrigen sieben Aufsätzen, die dem Themenbereich der ökonomischen Evaluation von Gesundheitsleistungen zugeordnet werden können. Sie befassen sich mit Problemen der Evaluationsmethodik und Ergebnisverwertung sowie mit Übersichten über Krankheitskostenstudien und Kosteneffektivitätsanalysen in ökonomisch bedeutsamen Krankheitsgebieten.

J.-M. Graf von der Schulenburg kritisiert aus wissenschaftlicher Perspektive, dass die gesundheitsökonomische Evaluation in Deutschland bisher praktisch keine Bedeutung bei Allokations- und Preisfindungsentscheidungen hat. Er bemängelt, dass die in den letzten Jahren dazu geführte Diskussion kaum Bezug auf die Erkenntnisse der wissenschaftlichen Forschung genommen hat. Er empfiehlt eine interdisziplinäre Auseinandersetzung mit den methodischen Grundlagen der ökonomischen Evaluation und ihre konsequente Anwendung in den Entscheidungsprozessen im Gesundheitswesen.

J. Schreyögg und T. Stargardt befassen sich mit den Möglichkeiten zur Durchführung gesundheitsökonomischer Evaluationen auf Grundlage von GKV-Routinedaten in Deutschland. Sie zeigen, dass Krankenkassen mittlerweile über Versichertendaten aus fast allen Sektoren des Gesundheitswesens verfügen, vergleichen die Charakteristika von Routinedaten mit denen von Primärdaten hinsichtlich einer Nutzung für gesundheitsökonomische Evaluation und geben einen Überblick über bisher durchgeführte gesundheitsökonomische Studien auf Basis von GKV-Routinedaten.

H. Leicht und H.-H. König berichten aktuelle Studienergebnisse zu Krank- heitskosten bei Demenz. Studienübergreifend geht der weitaus größte Teil der Kosten auf den erhöhten Pflegebedarf von Demenzpatienten zurück, der bei zu Hause lebenden Patienten hauptsächlich durch Angehörige gedeckt wird. Aufgrund des steigenden Pflegebedarfs steigen die Kosten mit dem Schweregrad der Demenz stark an. Die Kosten der medizinischen Versorgung machen hingegen nur einen kleinen Teil der Gesamtkosten aus und sind weitgehend unabhängig vom Schweregrad.

Der Beitrag von T. Lehnert und H.-H. König behandelt gesundheitsökonomische Aspekte der Multimorbidität bei älteren Patienten. Hierfür wurden empirische Studien der internationalen Literatur systematisch ausgewertet und zusammengefasst. Fast alle Studien fanden einen Zusammenhang zwischen der Anzahl an chronischen Erkrankungen und der Inanspruchnahme von Versorgungsleistungen und/oder den Versorgungskosten. Vor diesem Hintergrund diskutieren die Autoren wichtige Implikationen für die Forschung und Versorgung von älteren Menschen mit Mehrfacherkrankungen.

T. Reinhold, F. Müller-Riemenschneider, D. McBride, B. Brüggenjürgen und S.N. Willich zeigen am Beispiel der Behandlung der koronaren Herzerkrankung mittels medikamentenbeschichteter Stents, dass die in der internationalen Literatur vorliegenden Kosteneffektivitätsanalysen kaum ausreichende Evidenz für Allokationsentscheidungen im deutschen Gesundheitswesen liefern können. Daraus leiten sie die Forderung nach zusätzlichen langfristigen und sektorübergreifenden Evaluationsstrategien ab, für die Routinedaten eine mögliche Datenbasis darstellen können.

W.H. Rogowski, S.D. Grosse, E. Meyer, J. John und S. Palmer illustrieren am Beispiel des Screenings auf hereditäre Hämochromatose die Methode der Berechnung des Erwartungswerts perfekter Information. Besteht Unsicherheit bezüglich der Kosteneffektivität, ermöglicht diese Methode eine Abschätzung, ob sich die Investition in zusätzliche Forschung zur Wirksamkeit und Wirtschaftlichkeit lohnt. Im vorliegenden Beispiel erweist sich der maximal 
erreichbare Nutzen weiterer Forschung als gering.

Der abschließende Beitrag von B. Brüggenjürgen, L. Kornbluth, J.V. Ferrara und S.N. Willich befasst sich mit der Evaluation personalisierter Therapien. Die Autoren zeigen, dass herkömmliche gesundheitsökonomische Evaluationen in diesem innovativen Therapiebereich nur schwer umzusetzen sind, und schlagen hinsichtlich der Erstattung solcher Behandlungsformen die Vereinbarung kreativer Risk-Sharing-Ansätze zwischen Kostenträgern und Herstellern vor.

Wir wünschen den Leserinnen und Lesern interessante Einblicke in die gesundheitsökonomische Forschung.

Ihre

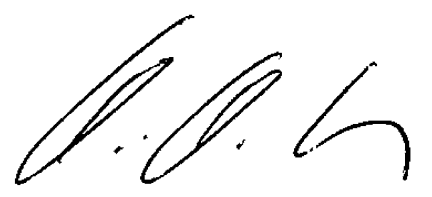

Hans-Helmut König

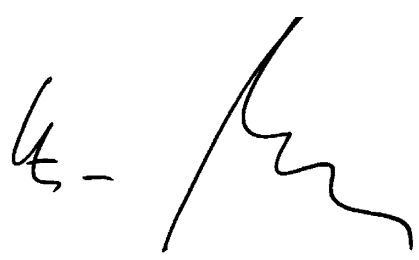

Stefan N. Willich

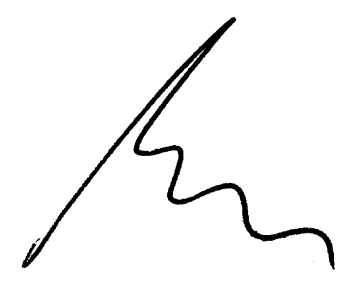

Uwe Koch-Gromus
Korrespondenzadresse

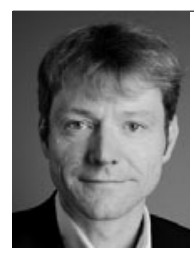

Prof. Dr. H.-H. König

Institut für Medizinische Soziologie, Sozialmedizin und Gesundheitsökonomie (IMSG), Hamburg Center for Health Economics, Universitätsklinikum Hamburg-Eppendorf Martinistr. 52, 20246 Hamburg h.koenig@uke. uni-hamburg.de

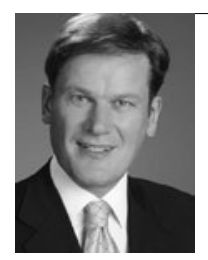

Prof. Dr. S.N. Willich Institut für Sozialmedizin, Epidemiologie und Gesundheitsökonomie, Charité Universitätsmedizin Berlin 10098 Berlin stefan.willich@charite.de

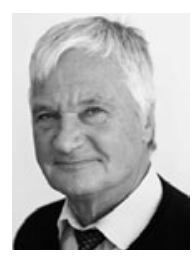

Prof. Dr. med. Dr. phil. U. Koch-Gromus Institut und Poliklinik für Medizinische Psychologie,

Zentrum für Psychosoziale Medizin, Universitätsklinikum Hamburg-Eppendorf Martinistr. 52, 20246 Hamburg Koch@uke.de

Bundesgesundheitsblatt - Gesundheitsforschung - Gesundheitsschutz $5 \cdot 2012$

603 